A paper submitted to work in prouress session at $9^{\text {th }}$ AIAA/ISSMO Symposium on Multidisciplinary Analysis and Optimization, Atlanta Ga, Sept 2072.

\title{
USE OF HIGH FIDELITY METHODS IN MULTIDISCIPLINARY OPTIMIZATION - A PRELIMINARY SURVEY
}

\author{
Guru P. Guruswamy \\ NASA Ames Research Center, Moffett Field, CA 94035-1000 \\ $\mathrm{Ph}(650) 604$ 6329, Fax : (650) 604 1095, email: gguruswamy@ mail.arc.nasa.gov
}

\begin{abstract}
Multidiciplinary optimization is a key element of design process. To date multidiscipline optimization methods that use low fidelity methods are well advanced. Optimization methods based on simple linear aerodynamic equations and plate structural equations have been applied to complex aerospace configurations. However, use of high fidelity methods such as the Euler/Navier Stokes for fluids and 3-D finite elements for structures has begun recently. As an activity of Multidiscipline Design Optimization Technical Committee (MDO TC) of AIAA an effort was initiated to assess the status of the use of high fidelity methods in multidisciplinary optimization. Contributions were solicited through the members MDO TC committee. This paper provides a summary of that survey.
\end{abstract}

\section{INTRODUCTION}

Multidisciplinary optimization is becoming important for aerospace structures mostly to address aeroelastic issues and weight reduction. Aeroelasticity that involves strong coupling of fluids, structures and controls is an important element in designing an aircraft. Computational aeroelasticity based on low fidelity methods such as the linear aerodynamic flow equations coupled with the modal structural equations, are well advanced. Although these low fidelity approaches are computationally less intensive, they are not adequate for the analysis of configurations which can experience complex flow/structure interactions. For example, supersonic transports can experience vortex induced aeroelastic oscillations whereas subsonic transports can experience transonic buffet associated structural oscillations[1]. Both aircraft may experience a dip in flutter speed at the transonic regime. Vortical tail of F18A experienced structural oscillations due to unsteady vortical flow[2]. An abrupt wing-stall phenomenon associated with structural motions was observed for F18E/F[3]. The X-34 launch vehicle experienced aeroelastic instability at low supersonic speeds[4]. Excess weight became a crucial design issue for X-33. For all these cases it was found that current analysis and design methods based on low fidelity methods were not adequate. In order to avoid undesirable aeroelastic behavior, multidisciplinary optimization is needed in aeroelastic design process. Current high fidelity method used for fluids is the Euler/Navier -Stokes (ENS) equations based finite difference and that for structures is the Lagrange equation based finite element (FE). Using these high fidelity methods, optimization can be done to avoid undesirable aeroelastic behaviors and minimize weight. 
Multidisciplinary optimization involves many aeroelastic computations. Aeroelastic computations are typically orders of magnitude more expensive than steady calculations on rigid configurations because of multidisciplines that add additional complexities from physics. Figure 1 shows a typical growth in the requirement of computational time to compute a single aeroelastic response for different geometric complexities. All CPU hrs required are presented in terms of a C-90 single processor. The growth in CPU time required is exponential. Thousands of such computations are required for a single aircraft design. Advances in parallel computers have made such computations more feasible[5].

Fluids and structural domairs can be modeled at various levels of complexity both in physics and geometry. For design, aerodynamic data may be used at several levels of fidelity starting from the low-fidelity look-up tables to the high fidelity Navier-Stokes solutions. Similarly for structures, the data can be obtained starting from the low fidelity assumed shape functions to detailed threedimensional finite elements. As the fidelity of modeling increases, it becomes more difficult to handle complex geometry. Figure 2 illustrates the typical levels of modeling complexities involved both for fluids and structures. In this survey levels of fidelity illustrated in Fig 2 is taken as a road map to assess the status of the use of high fidelity methods in multidisciplinary optimization.

\section{APPROACH}

a) Send out questioner to those who are conducting active research in MDO. Most of the contacts were made through about 40 MDOTC members who represent a broad spectrum researchers.

b) Accept responses that has objective evidence such as archived paper/report or url.

c) Tabulate the received information and assess a fidelity/complexity index.

d) Place the information on MDO TC home page

e) Write a summary paper

\section{QUESTIONEER SENT OUT}

As an activity under the applications sub-committee of the AIAA MDO technical committee we plan to conduct a survey to find the status of MDO applications using high fidelity methods. Please send information (publication details ) about work you have done in related area. An electronic version of full report/paper (MS Word, PDF, HTML) is appreciated. Please forward this message to others who may be working in this or related fields.

Requirements for the information to be included in literature survey.

1. Minimum 2 disciplines

2. Unclassified/non-proprietary/Public Domain 
Please indicate the level of fidelity of discipline modeling and optimization method. Following is a guideline

Fluids : (Include the type of configuration and grid size where applicable)

1. Navier Stokes

2. Euler

3. Full potential

4. TSP

5. Linear

6. Empirical/Other (Specify)

Structures : (Include the type of configuration and number of elements where applicable)

1. 3D FEM

2. 2D FEM

3. Equivalent Plate

4. Modal

5. Shape Functions

6. Empirical/Other (Specify)

Controls :

1. Time Domain Feed Back

2. Frequency Domain

3. Empirical/Other (Specify)

Propulsion

1. 3D Navier Stokes

2. 2 D Navier Stokes

3. 1D Navier Stokes

4. Empirical

Optimization Methods.

1. Gradient Method

a) Adjoint Method

b) other

1. Evolutionary Algorithm

a) GA

b)other

2. Other (e.g. Physical programming) 


\section{GUIDELINE FOR COMPUTING COMPLEXITY/FIDELITY INDEX}

It is assumed that the complexity of the problem is represented by the grid size used for modeling the flow and structures. Indices for various disciplines selected are shown below.

\section{FIDELITY INDEX}

FLUIDS
a) Navier-Stokes (10)
b) Euler (5)
c) Full Potential (4)
d) Linear/Panel (2)
e) Empirical/Tables(1)

COMPLEXITY INDEX

Fluids : Number of Grid points in 100K

Structures : Number of elements in 1000.
STRUCTURES

a)3D Nonlinear FEM (10)

b)3-D FEM (7)

c)2D FEM (5)

d) 1-D FEM, Modal (3)

e) Shape Functions (1)

\section{CONTROL}

a)Time Domain(10)

c) Frequency Domain(6) b) 2-D NS (6)

c)1-D NS (4)

d)Empirical(1)

\section{RESULTS}

To date about 20 responses were received. Out of them about 8 satisfied he requirements of the survey. A preliminary table 1 is created based on the responses. From table 1 it is seen that the highest fidelity for fluids is the Navier-Stokes equations. Structures is still limited to low fidelity models such as beam models. The traditional optimization approaches based on the gradient method are still in use. The use of evolutionary algorithm that may have advantage for the multiobjective multidisciplinary applications are becoming more popular. More details will be provided in the final paper.

\section{REFERENCES}

1. Dobbs, S. K. and Miller, G. D.: Self-Induced Oscillation Wind Tunnel Test of a Variable Sweep Wing. AIAA Paper 85-0739, Apr. 1985.

2. Findlay, D. :Numerical Analysis of Vertical Tail Buffet. AIAA 97-0621, Reno 1997

3. Grove, D., Liaosa, T, Woodson, S and Stookesberry, D. : Computational Fluid Dynamics Study of an Abrupt Wing Stall Phenomena on an F/A-18E. AIAA-2002-1025, Reno Jan 2002.

4. Blades, E.L, Ruth, M. and Furmann, H.D: Aeroelastic Analysis of the X-34 Launch Vehicle. AIAA-99-1352. April 1999. 
5. Guruswamy, G.P : Impact of Parallel Computing On High Fidelity Based Multidiciplinary Analysis. AIAA $98-4709$

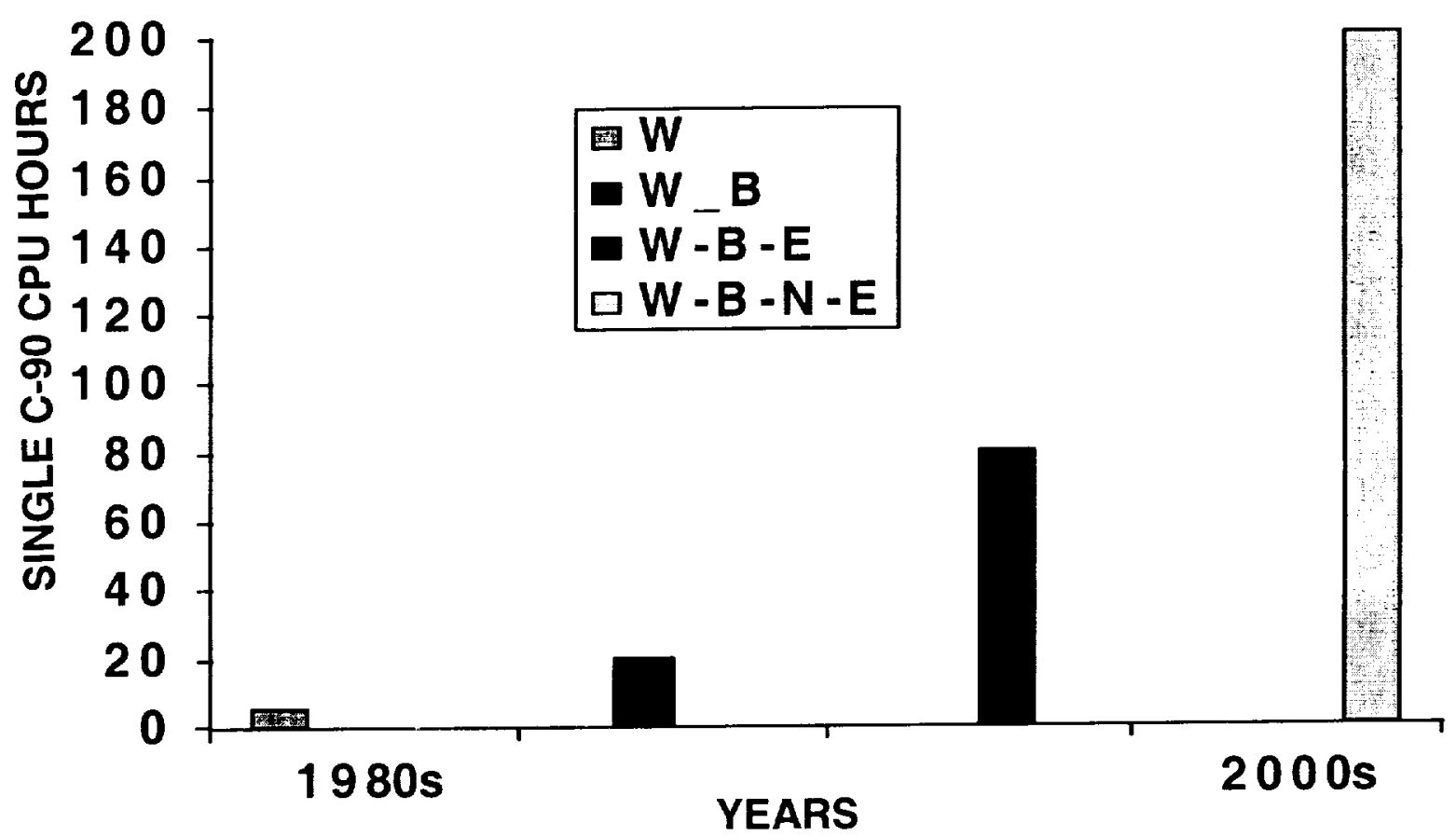

Fig. 1 CPU Hrs needed for a typical aeroelastic computation using coupled Navier-Stokes and modal equations.
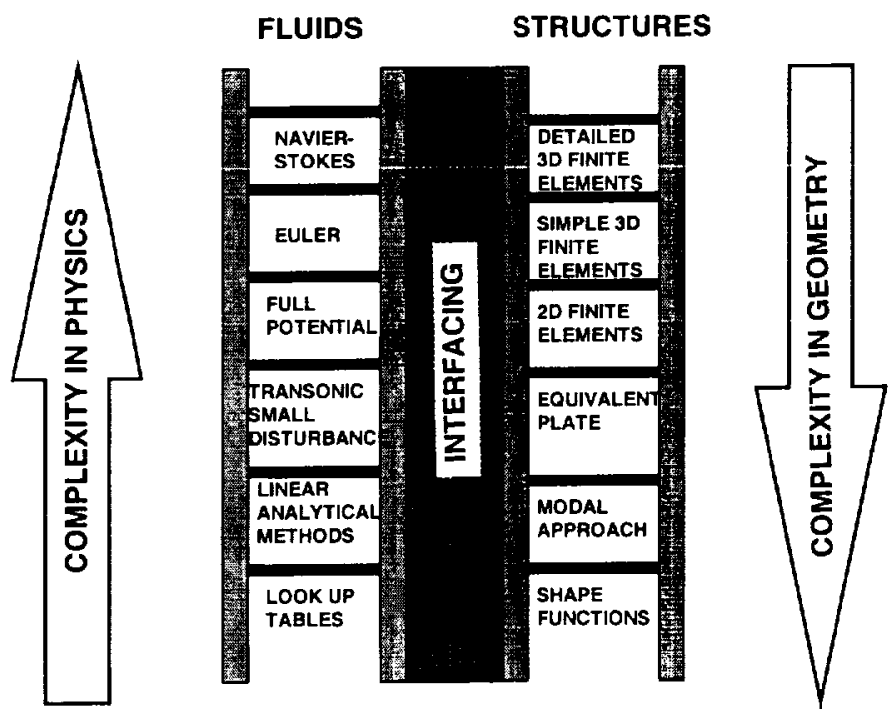

Figure 2. Varying levels of fidelity in modeling for fluids and structures. 


\begin{tabular}{|c|c|c|c|c|c|}
\hline POC & $\begin{array}{l}\text { FLUIDS/ } \\
\text { GRID }\end{array}$ & $\begin{array}{l}\text { STRUCTURES } \\
\text { /ELEMENTS }\end{array}$ & $\begin{array}{l}\text { OTHER } \\
\text { PROP/CONR }\end{array}$ & OPTIMIZATION & $\begin{array}{l}\text { F-C } \\
\text { INDEX }\end{array}$ \\
\hline RAVEH & EULER/600K & W-B FEM/1K & & GRADIENT & 14 \\
\hline HOLST & FP/100K & BEAM & & GA & 8 \\
\hline KNILL & EULER/500K & Plate & & vC & 12 \\
\hline OYAMA & NS/500K & BEAM & & GA & 17 \\
\hline GIUNTA & EULER/300K & PLATE/1K & & GRADIENT & 11 \\
\hline BLAIR & PANEL & W-B NONLINEAR & & $\mathbf{P P}$ & 9 \\
\hline ZANG & EULER/400K & 3D FEM/2K & & CONMIN & 18 \\
\hline KIM & EULER/100K & 2D FEM & & GA & 11 \\
\hline RODRIGUEZ & NS/500K & & 1-D NS PROP & NPSOL & 19 \\
\hline
\end{tabular}

GA : Genetic algorithm, VC : Variable Complexity, PP : Physical Programming,

F-C : Preliminary Fidelity-Complexity Index

Rodriguez: AIAA 2000-4875

Holst : Jl of Aircraft Vol 35, No 3 1998, pp412

Raveh : Jl of Aircraft, Vol 36, No 6, 1999, pp1007-1015

Oyama: ECCOMAS 2000, Bracelona, Sept 2000

Giunta : J of Aircaft Design, Vol 2, No 4, 1999 pp 207-230.

Blair : AIAA-2002-1337

Messaic : www.rpi.edu/ messac

Zang : url TBD 